\title{
HOT TOPICS
}

\section{EEG microstate sequences suggest abnormally chaotic brain dynamics in psychosis}

\author{
Michael Murphy (iD ${ }^{1,2}$ and Dost Öngür ${ }^{1,3}$ \\ Neuropsychopharmacology (2021) 46:223-224; https://doi.org/10.1038/s41386-020-00800-x
}

Psychiatric disorders are associated with abnormal patterns of brain connectivity. Characterization of brain connectivity in humans is complicated by the spatial and temporal resolution of neuroimaging techniques, which partition the brain into artificial units. Furthermore, connectivity is dynamic-brain regions become coupled and uncoupled over time. To understand the role of brain connectivity in psychiatric illness, we need a way of characterizing connectivity that makes minimal assumptions about functional units and can quantify patterns of changes in connectivity (Fig. 1).

Recently, there has been interest in the use of electroencephalographic (EEG) microstates to study brain networks [1]. Micro- states are characteristic, semi-stable voltage topographies. During an EEG recording, a specific topography, or "microstate", persists for several milliseconds and then transitions to a different microstate. A small number of microstates are needed to explain most of the variance in the EEG signal. Microstate topographies are data-driven and yet four canonical microstate topographies ( $A$, $B, C$, and D) have been replicated in multiple studies.

Microstates are generated by neural assemblies. Source modeling studies, which employ anatomical and biophysical constraints to model cortical activity from EEG data, suggest that these neural assemblies overlap with, but are distinct from, functional magnetic
A
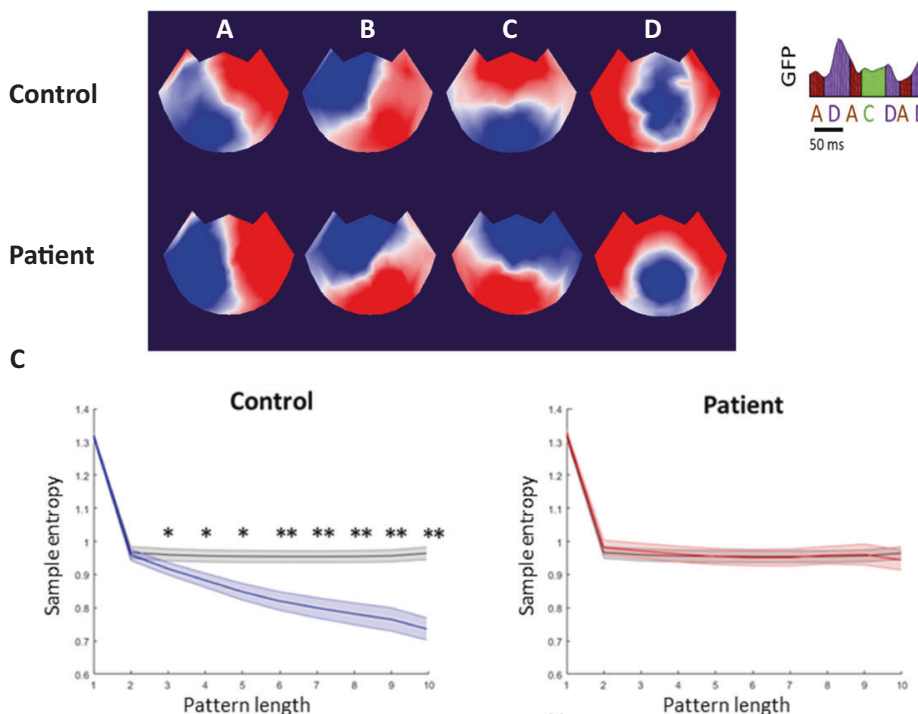

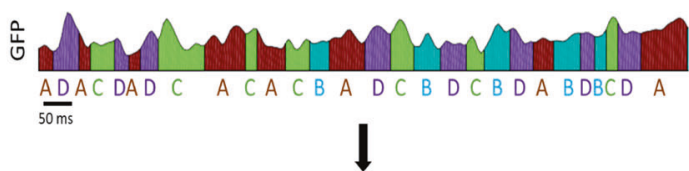

ADACDADCACACBADCBDCBDABDBCDA

D

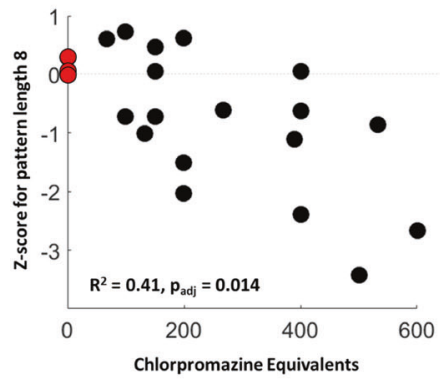

Fig. 1 Adapted from [6]. a Microstate topographies from controls and patients with early-course psychosis. b Microstate sequences generated from microstate-fitted EEG data. GFP global field power. $\mathbf{c}$ Pattern length $(m=1: 10)$ vs. average entropy across participants. The left panel shows control data (blue curve, shaded region is standard error of the mean) and random permutations of control data (black line and black shaded region). The right panel shows patient data (red curve and red shaded region) and random permutations of patient data (black line and black shaded region). ${ }^{*} p<0.01,{ }^{* *} p<0.001$. d Scatter plot showing the relationship between CPZ equivalents (in milligrams) and entropy $z$ scores for pattern length $m=8$. Lower $z$ scores indicate more structured, less chaotic, transition patterns. Note that the three unmedicated patients (red circles) all had $z$ scores close to zero.

\footnotetext{
${ }^{1}$ Department of Psychiatry, Harvard Medical School, Belmont, MA, USA; ${ }^{2}$ McLean Hospital, Belmont, MA, USA and ${ }^{3}$ Schizophrenia and Bipolar Disorder Research Program, McLean Hospital, Belmont, MA, USA

Correspondence: Michael Murphy (mmurphy@mgh.harvard.edu)
}

Published online: 14 August 2020 
resonance imaging (fMRI) resting state networks [2]. Microstate topography is a spatially blurred view of these underlying assemblies while microstate duration provides information about the ability of the brain to sustain large scale connectivity. Microstates and microstate transitions are most likely driven by the activity of the cortico-thalamocortical network hubs, which drive the alpha rhythm [2]. Individual microstates have been associated with fMRI networks, for example, microstates $C$ and $D$ with salience and attention networks, respectively [3]. However, it is likely that fMRI networks are temporally smoothed representations of cortical activity underlying sequences of microstates [4].

Previous work has demonstrated microstate abnormalities in individuals with schizophrenia [5]. In a recent study, we expanded upon this work by using sample entropy, a measure of complexity, to study sequences of microstates in individuals with early-course psychosis [6]. We compared the entropy of the microstate sequences for each subject with the entropy of randomized sequences of conditioned on the distribution of microstate labels. We studied sequences up to length 10 (roughly $0.5 \mathrm{~s}$ ) because this allowed us to quantify the complexity of the dynamics of brain networks at a time scale that is inaccessible to fMRI.

In control participants, at longer scales, sample entropy decreased. This means that microstate sequences are self-similar across multiple scales. Specific sequences of pairs of microstates transitioning between each other were overrepresented. In patients with early-course psychosis, we found no such decreases in sample entropy. Entropy was unrelated to symptom burden, however, entropy was the highest in medication-free individuals and was negatively correlated with antipsychotic dose (measured as chlorpromazine equivalents (CPZ)). Therefore, patients with psychotic disorders have abnormally random patterns of transition between brain networks and one effect of medication may be to restore structure to these transitions. Future longitudinal studies could provide further evidence for this. The study of microstate sequences permits the characterization of cortico-thalamocortical networks at a previously inaccessible temporal resolution, which could reveal novel state and trait biomarkers of psychiatric illness [7].
FUNDING AND DISCLOSURE

This work was funded by a National Alliance for Research in Schizophrenia and Affective Disorders Young Investigator Grant from the Brain and Behavior Research Foundation (Grant no. 27017 [to MM]), by National Institute of Mental Health Grant K23 MH118565 (to MM), and by National Institute of Mental Health Grant K24 MH104449 (to DO). The authors have no potential conflicts of interest.

\section{AUTHOR CONTRIBUTIONS}

$\mathrm{MM}$ and DO wrote the paper.

\section{ADDITIONAL INFORMATION}

Publisher's note Springer Nature remains neutral with regard to jurisdictional claims in published maps and institutional affiliations.

\section{REFERENCES}

1. Michel CM, Koenig T. EEG microstates as a tool for studying the temporal dynamics of whole-brain neuronal networks: a review. Neuroimage. 2018;180:577-93.

2. Milz P, Pascual-Marqui RD, Achermann P, Kochi K, Faber PL. The EEG microstate topography is predominantly determined by intracortical sources in the alpha band. Neuroimage. 2017;162:353-61.

3. Britz J, Van De Ville D, Michel CM. BOLD correlates of EEG topography reveal rapid resting-state network dynamics. Neuroimage. 2010;52:1162-70.

4. Milz $P$, Faber PL, Lehmann D, Koenig T, Kochi K, Pascual-Marqui RD. The functional significance of EEG microstates-associations with modalities of thinking. Neuroimage. 2016;125:643-56.

5. Rieger K, Hernandez LD, Baenninger A, Koenig T. 15 years of microstate research in schizophrenia - Where are we? A meta-analysis. Front Psychiatry. 2016;7:1-7.

6. Murphy M, Stickgold R, Öngür D. Electroencephalogram microstate abnormalities in early-course psychosis. Biol Psychiatry Cogn Neurosci Neuroimaging. 2020;5:35-44.

7. Murphy $M$, Whitton $A E$, Deccy $S$, Ironside $M L$, Rutherford $A$, Beltzer $M$, et al. Abnormalities in electroencephalographic microstates are state and trait markers of major depressive disorder. Neuropsychopharmacology. 2020:1-8. https://doi. org/10.1038/s41386-020-0749-1 